\title{
Simple undergraduate experiment for synthesizing and analyzing non-uniformly polarized beams by means of a Fresnel biprism
}

Gemma Piquero, Ismael Marcos-Muñoz, and J. C. G. de Sande

Citation: American Journal of Physics 87, 208 (2019); doi: 10.1119/1.5089423

View online: $\mathrm{https}: / /$ doi.org/10.1119/1.5089423

View Table of Contents: https://aapt.scitation.org/toc/ajp/87/3

Published by the American Association of Physics Teachers

\section{ARTICLES YOU MAY BE INTERESTED IN}

Spectroscopy of neon for the advanced undergraduate laboratory

American Journal of Physics 87, 223 (2019); https://doi.org/10.1119/1.5088806

Simple analytic expressions of Fresnel diffraction patterns at a straight strip and slit for Gaussian wave illumination

American Journal of Physics 87, 171 (2019); https://doi.org/10.1119/1.5089415

Kepler and the origins of the theory of gravity

American Journal of Physics 87, 176 (2019); https://doi.org/10.1119/1.5089751

From measuring electron charge to exploring particle-wave duality: A new didactic experimental approach American Journal of Physics 87, 194 (2019); https://doi.org/10.1119/1.5086392

Elastic collisions of smooth spherical objects: Finding final velocities in four simple steps

American Journal of Physics 87, 200 (2019); https://doi.org/10.1119/1.5089753

Unusual broadening of wave packets on lattices

American Journal of Physics 87, 186 (2019); https://doi.org/10.1119/1.5089752

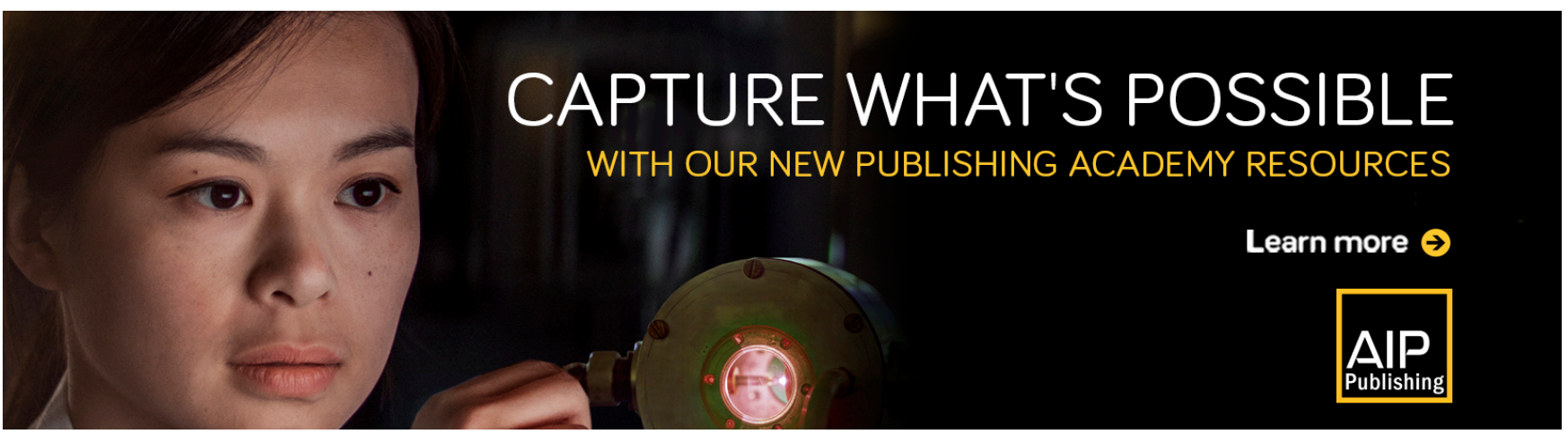




\title{
Simple undergraduate experiment for synthesizing and analyzing non-uniformly polarized beams by means of a Fresnel biprism
}

\author{
Gemma Piqueró $^{\text {a) }}$ and Ismael Marcos-Muñoz \\ Departamento de Óptica, Fac. CC. Físicas, Universidad Complutense de Madrid, 28040 Madrid, Spain \\ J. C. G. de Sande \\ ETSIS de Telecomunicación, Universidad Politécnica de Madrid, Campus Sur, 28031 Madrid, Spain
}

(Received 13 September 2018; accepted 18 January 2019)

\begin{abstract}
A simple scheme to synthesize non-uniform patterns of polarization across the transverse section of a beam is proposed with the standard materials in an undergraduate optics laboratory. The experiment is based on the superposition of two orthogonally polarized fields obtained by using a Fresnel biprism and dichroic polarizers. Although no interference pattern appears in the superposition area, a non-uniformly totally polarized field is synthesized. Analytic expressions for the Jones vector and Stokes parameters of the output beam are calculated and, in the process, students can cement their knowledge about the representation of polarized light with these formalisms. The experimental polarization pattern is either obtained from intensity measurements with a CCD camera or measured directly with a commercial polarimeter modified with a pinhole. This experiment will help students discover an easy way to vary the state of polarization across the transverse section of a light field. (c) 2019 American Association of Physics Teachers.

https://doi.org/10.1119/1.5089423
\end{abstract}

\section{INTRODUCTION}

Generally, in undergraduate physics courses, interference of light is treated within the classical theory of light under the scalar approximation. One of the first examples introduced is Young's double slit experiment. ${ }^{1}$ This is one of the most important experiments in physics since it plays a crucial role in the development of optics, the understanding of the nature of light, the comprehension of quantum mechanics, and the foundation of the theory of coherence. An example of an analogous interferometer is the Fresnel biprism, which presents some advantages with respect to the doubleslit experiment, for instance, the reduction of light losses and the possibility to produce interference with spherical or plane waves. ${ }^{1}$ When the topic of interference is being taught in physics courses, questions that typically arise are what would be observed if the superposing beams are orthogonally polarized to each other and what would happen if a third polarizer is placed after the slits. Unfortunately, questions about the state of polarization in the superposition area of the two beams are usually discarded.

On the other hand, the topic of polarization of light is also present in the syllabus of optics courses. One studies how to describe, detect, and modify the polarization, but always considering a uniformly polarized beam across the transverse section. However, optical beams can be non-uniformly polarized. In this work, we will combine the two concepts of interference and polarization of light, focusing on the synthesis and analysis of a non-uniformly totally polarized (NUTP) field.

The synthesis, characterization, and propagation of paraxial NUTP beams in their transverse section have received extraordinary attention among the scientific community in recent years. $^{2-11}$ The usefulness of NUTP beams in the improvement of numerous applications has been largely demonstrated. To cite some examples, NUTP beams have been used in particle manipulation, ${ }^{12,13}$ polarimetry, ${ }^{14-16}$ material processing, ${ }^{17-19}$ angular momentum generation, ${ }^{20-22}$ optical encryption, ${ }^{23}$ etc. Numerous methods for synthesizing NUTP beams have been proposed..$^{5,6,10,11,24,25}$ One straightforward way to obtain them is through superposition of two beams presenting different states of polarization. Moreover, interference with polarized light allows one to obtain relevant information about its polarization and coherence characteristics. ${ }^{26-34}$

In addition, numerous works deal with the interference of polarized light from a didactic point of view. Some of them are focused on the experimental confirmation of the FresnelArago laws with Young's double slit experiment. ${ }^{35-40}$ In others, different experimental schemes have been proposed to study interference with polarized light, for example, by using a Michelson interferometer, anisotropic prisms, or spatial light modulators. ${ }^{41-45}$ In some of these papers, theoretical polarization maps have been shown. However, from the experimental point of view, only a single polarizer has been used to test the non-uniformity of the polarization state across the output beam section.

In this work, the issue of non-uniformly polarized beams is presented to students through a simple and inexpensive interference experiment using a Fresnel biprism where two dichroic polarizers are placed before each part of the biprism. The transmission axes are oriented perpendicular to each other so two orthogonally polarized fields are generated. After the biprism, these two fields superpose, and simple analytic expressions for the Jones and Stokes vectors of the output beam are derived. The students can explore two alternative ways of measuring the polarization state and verify that the theoretical results fit very well with the experimental results. The polarization state pattern is determined by means of either a CCD camera or a commercial polarimeter.

The work has been organized as follows. In Sec. II, the underlying theory is presented. The two experimental setup and results are discussed in Sec. III. Finally, Sec. IV summarizes the main conclusions of this paper.

\section{THEORY}

The Jones formalism is an appropriate tool to deal with totally polarized light. ${ }^{1,46}$ The electric field of a monochromatic, totally uniformly polarized plane wave propagating 
along the $z$ axis of a suitable reference system can be expressed as

$$
\mathbf{E}_{\text {in }}=E_{0 x} \cos (\omega t-k z) \mathbf{u}_{x}+E_{0 y} \cos (\omega t-k z-\varphi) \mathbf{u}_{y},
$$

where the subscript "in" indicates incident beam, $E_{0 x}$ and $E_{0 y}$ are the amplitudes of the field along the $x$ and $y$ axes, respectively, $\varphi$ is the relative phase between both field components, $\omega$ is the angular frequency, $t$ is the time, $k$ is the wavevector, and $\mathbf{u}_{x}\left(\mathbf{u}_{y}\right)$ is a unit vector along $x(y)$ direction. The two orthogonal components of the corresponding electric field can be arranged in a $2 \times 1$ Jones vector

$$
\mathbf{E}_{\mathrm{in}}=\left(\begin{array}{c}
E_{0 x} \\
E_{0 y} e^{-i \varphi}
\end{array}\right),
$$

where $i$ denotes the imaginary unit and Euler's relation has been used.

This beam impinges onto a Fresnel biprism of maximum thickness $D$ and acute angle $\alpha$ (Fig. 1). Two dichroic polarizers, $\mathrm{P}_{1}$ and $\mathrm{P}_{2}$, with perpendicular transmission axes, have been placed in front of the top and bottom prisms, respectively, so light passing through the top prism has an orthogonal polarization state to that passing through the bottom prism. In this case, no interference fringes are formed at the observation plane due to the orthogonality of the two fields superposing at any point. Multiple reflections between the polarizers and the biprism are neglected. The two orthogonal fields observed at a given point (for example, at a height $y_{0}$ in the observation plane) have acquired different phases due to the difference in the optical paths $n d\left(y_{1}\right)+l_{1}$ and $n d\left(y_{2}\right)+l_{2}$, respectively. Here, $n$ is the refractive index of the biprism, $d\left(y_{j}\right)$ with $j=1,2$ is the propagation distance inside the biprism for different $y_{j}$, and $l_{j}$ is the propagation distance outside the biprism (see Fig. 1). Due to the variation of the optical path difference, the polarization state at the observation plane changes with height $y_{0}$ and the field so obtained is non-uniformly polarized.

The field at an arbitrary point in the observation plane can be expressed as

$$
\mathbf{E}_{\mathrm{op}}=\left(\begin{array}{c}
t_{\perp} t_{\perp}^{\prime} E_{0 x} e^{-i k\left[n d\left(y_{1}\right)+l_{1}\right]} \\
t_{\|} t_{\|}^{\prime} E_{0 y} e^{-i \varphi} e^{-i k\left[n d\left(y_{2}\right)+l_{2}\right]}
\end{array}\right),
$$

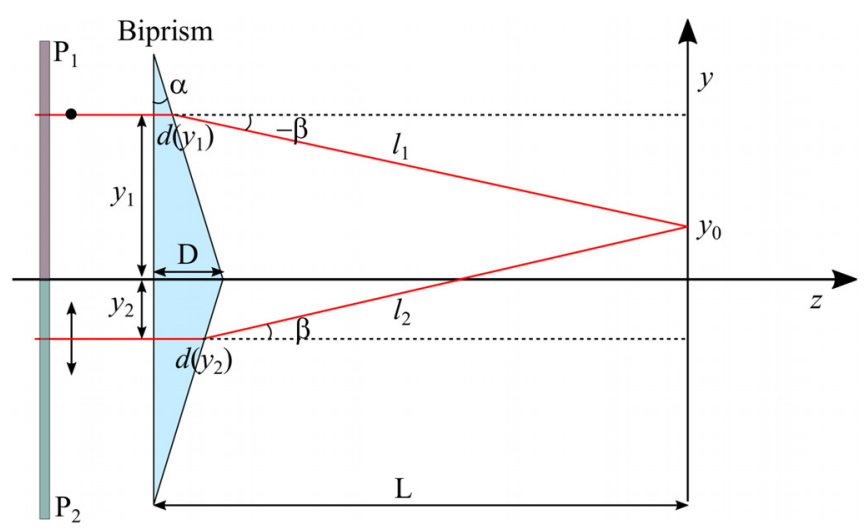

Fig. 1. Schematic of a Fresnel biprism together with the incident and output rays reaching the same observation point $y_{0}$. where the subscript "op" stands for observation plane, $t_{\perp}$ and $t_{\perp}^{\prime}\left(t_{\|}\right.$and $\left.t_{\|}^{\prime}\right)$ are the transmission coefficients at the input and output surfaces of the biprism for the field component orthogonal (parallel) to the incidence plane. The propagation direction of the upper (lower) part of the beam after the biprism forms a small angle $-\beta(\beta)$ with the $z$ axis, so the parallel component to the incidence plane is not exactly parallel to the $y$ axis. However, this has a negligible effect on the state of polarization. Due to the opposite deviation of the exiting beams relative to the $z$ axis, the two output beams superpose in an extended but finite region behind the biprism. The extent of such a region depends on the beam width and the prism angle $\alpha$.

The propagation distance inside the biprism is related to the biprism angle and maximum thickness $D$ through (Fig. 1)

$$
d(y)=D-|y| \tan \alpha .
$$

From Snell's law of refraction and the geometry of the experiment (Fig. 1), the optical paths can be expressed in terms of experimental quantities $L$ (distance from the input plane of the biprism to the observation plane), $D$, and $\alpha$. The angle $\beta$ in Fig. 1 can be found by applying Snell's law

$$
n \sin \alpha=\sin (\alpha+\beta) \text {. }
$$

On the other hand, once the height $y_{0}$ on the observation plane is fixed, the heights $y_{1}$ and $y_{2}$ can be found as

$$
y_{0}=y_{1}-l_{1} \sin \beta=y_{2}+l_{2} \sin \beta,
$$

which allows us to express $y_{1}$ and $y_{2}$ in terms of $y_{0}$

$$
\begin{aligned}
& y_{1}=y_{0}+l_{1} \sin \beta, \\
& y_{2}=y_{0}-l_{2} \sin \beta .
\end{aligned}
$$

Substitution of Eq. (7) into Eq. (4) yields

$$
\begin{aligned}
& d\left(y_{1}\right)=D-\left(y_{0}+l_{1} \sin \beta\right) \tan \alpha, \\
& d\left(y_{2}\right)=D+\left(y_{0}-l_{2} \sin \beta\right) \tan \alpha .
\end{aligned}
$$

The angle $\beta$ in Fig. 1 is also related to the separation $L$ between the biprism input plane and the observation plane, and the distances $l_{1}$ and $d\left(y_{1}\right)$ or $l_{2}$ and $d\left(y_{2}\right)$ as

$$
\cos \beta=\frac{L-d\left(y_{1}\right)}{l_{1}}=\frac{L-d\left(y_{2}\right)}{l_{2}} .
$$

So, using Eq. (9), the following relations can be obtained for the distances $l_{1}$ and $l_{2}$ :

$$
\begin{aligned}
& l_{1} \cos \beta=L-d\left(y_{1}\right)=L-D+\left(y_{0}+l_{1} \sin \beta\right) \tan \alpha, \\
& l_{2} \cos \beta=L-d\left(y_{2}\right)=L-D-\left(y_{0}-l_{2} \sin \beta\right) \tan \alpha,
\end{aligned}
$$

which can be expressed in terms of experimental parameters as

$$
\begin{aligned}
& l_{1}=\frac{L-D+y_{0} \tan \alpha}{\cos \beta-\sin \beta \tan \alpha}, \\
& l_{2}=\frac{L-D-y_{0} \tan \alpha}{\cos \beta-\sin \beta \tan \alpha} .
\end{aligned}
$$

By using these values and the distances $d\left(y_{1}\right)$ and $d\left(y_{2}\right)$ obtained from Eq. (4), the optical paths become 


$$
\begin{aligned}
n d\left(y_{1}\right)+l_{1}= & n\left(D-y_{0} \tan \alpha\right) \\
& +\frac{L-D+y_{0} \tan \alpha}{\cos \beta-\sin \beta \tan \alpha}(1-n \sin \beta \tan \alpha), \\
n d\left(y_{2}\right)+l_{2}= & n\left(D+y_{0} \tan \alpha\right) \\
& +\frac{L-D-y_{0} \tan \alpha}{\cos \beta-\sin \beta \tan \alpha}(1-n \sin \beta \tan \alpha),
\end{aligned}
$$

so the optical path difference is

$$
\begin{aligned}
\Delta & \equiv n d\left(y_{2}\right)+l_{2}-n d\left(y_{1}\right)-l_{1} \\
& =2 y_{0} \tan \alpha\left(n-\frac{1-n \sin \beta \tan \alpha}{\cos \beta-\sin \beta \tan \alpha}\right) .
\end{aligned}
$$

The Jones vector of the field at the observation plane can then be expressed as

$$
\mathbf{E}_{\mathrm{op}}=\left(\begin{array}{c}
t_{\perp} t_{\perp}^{\prime} E_{0 x} \\
t_{\|} t_{\|}^{\prime} E_{0 y} e^{-i \varphi} e^{-i 2 k y_{0} \tan \alpha\left(n-\frac{1-n \sin \beta \tan \alpha}{\cos \beta-\sin \beta \tan \alpha}\right)}
\end{array}\right),
$$

where an irrelevant common phase factor has been omitted.

Under the paraxial approximation ( $\alpha$ is typically of the order of $1^{\circ}$ ), the simplified expressions $\tan \alpha \cong \sin \alpha \cong \alpha$, $\sin \beta \cong \beta \cong(n-1) \alpha$, and $\cos \beta \cong 1$ can be used, so the Jones vector of the field is approximately

$$
\mathbf{E}_{\mathrm{op}} \cong\left(\begin{array}{c}
t_{\perp} t_{\perp}^{\prime} E_{0 x} \\
t_{\|} t_{\|}^{\prime} E_{0 y} e^{-i \varphi} e^{-i 2 k y_{0} \alpha(n-1)}
\end{array}\right) .
$$

Note that Eq. (15) could be obtained by calculating the superposition of two plane waves propagating at angles $\pm \beta$ and applying the paraxial approximation. The calculated field at the observation plane represents a nonuniformly polarized field, which can be modified at will by changing the input beam characteristics. Figure 2 shows the obtained polarization pattern for the case of linearly polarized at $45^{\circ}$ input light. A biprism with $n=1.515$, $\alpha=1.5^{\circ}$, and wavelength $\lambda=632.8 \times 10^{-6} \mathrm{~mm}$ was used. A periodic variation of the state of polarization along the $y$ direction is observed, that is, a polarization grating ${ }^{14,47-49}$ is generated in a simple way. The period $\Lambda$ of the polarization state variation can be obtained by equating the phase difference to $2 \pi$, resulting in

$$
\Lambda \cong \frac{\lambda}{2 \alpha(n-1)},
$$

which is $\Lambda \simeq 23 \mu \mathrm{m}$ for the experimental values.

A useful way to describe the state of polarization is by means of Stokes parameters. The reason for employing Stokes parameters instead of the Jones vector in the following is that the former represent directly measurable quantities. ${ }^{1}$ The Stokes parameters can be obtained via ${ }^{1}$

$$
\mathbf{S}=\left(\begin{array}{c}
S_{0} \\
S_{1} \\
S_{2} \\
S_{3}
\end{array}\right)=\left(\begin{array}{c}
\left|E_{x}\right|^{2}+\left|E_{y}\right|^{2} \\
\left|E_{x}\right|^{2}-\left|E_{y}\right|^{2} \\
2 \operatorname{Re}\left\{E_{x} E_{y}^{*}\right\} \\
2 \operatorname{Im}\left\{E_{x} E_{y}^{*}\right\}
\end{array}\right),
$$

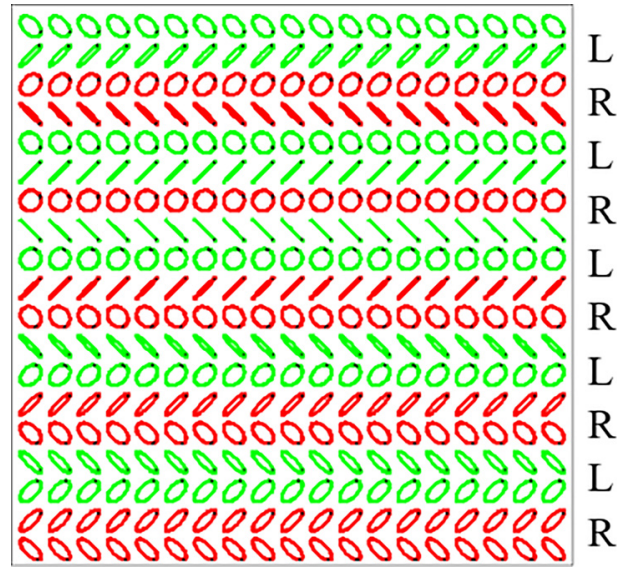

Fig. 2. Theoretical polarization pattern at the output of the biprism (with $n=1.515$ and $\alpha=1.5^{\circ}$ ) for input linearly polarized light with the azimuth at $45^{\circ}$, wavelength $\lambda=632.8 \mathrm{~nm}$. Labels $\mathrm{R}$ and $\mathrm{L}$ represent right-handed and left-handed polarized light, respectively.

where $\operatorname{Re}\{\cdot\}$ and $\operatorname{Im}\{\cdot\}$ denote real and imaginary part, respectively, and for brevity the dependence on the spatial coordinates has been omitted. The first Stokes parameter $\left(S_{0}\right)$ coincides with the total irradiance, while the three remaining parameters represent the differences between the contents of linearly polarized light along the $x$ and $y$ axes, between the contents of linearly polarized light at $45^{\circ}$ and $-45^{\circ}$, and between right-handed and left-handed circularly polarized contents of the beam, respectively. ${ }^{1}$

In our case, the Stokes vector of the field at the observation plane is

$$
\mathbf{S}=\left(\begin{array}{c}
\left|t_{\perp} t_{\perp}^{\prime} E_{0 x}\right|^{2}+\left|t_{\|} t_{\|}^{\prime} E_{0 y}\right|^{2} \\
\left|t_{\perp} t_{\perp}^{\prime} E_{0 x}\right|^{2}-\left|t_{\|} t_{\|}^{\prime} E_{0 y}\right|^{2} \\
2 t_{\perp} t_{\perp}^{\prime} t_{\|} t_{\|}^{\prime} E_{0 x} E_{0 y} \cos [2 k y \alpha(n-1)+\varphi] \\
2 t_{\perp} t_{\perp}^{\prime} t_{\|} t_{\|}^{\prime} E_{0 x} E_{0 y} \sin [2 k y \alpha(n-1)+\varphi]
\end{array}\right) .
$$

It can be easily checked that this set of Stokes parameters satisfy the relation $S_{0}^{2}=S_{1}^{2}+S_{2}^{2}+S_{3}^{2}$ in the whole observation plane, which means that the output beam is totally polarized. However, as the Stokes vector components $S_{2}$ and $S_{3}$ depend on the vertical coordinate, the field is a nonuniformly totally polarized beam. ${ }^{50}$

The polarization state can be graphically represented by its corresponding polarization ellipse, which can be directly obtained from the Stokes parameters. ${ }^{1,46}$ In fact, the azimuth $\psi$ and the ellipticity $\chi$ angles can be found as

$$
\tan 2 \psi=\frac{S_{2}}{S_{1}},
$$

and

$$
\tan 2 \chi=\frac{S_{3}}{\sqrt{S_{1}^{2}+S_{2}^{2}}},
$$

respectively. ${ }^{1,46}$

When the coordinate $y$ of the observation point varies, the state of polarization changes. Figure 2 shows the polarization pattern calculated at the output of a biprism with refractive 


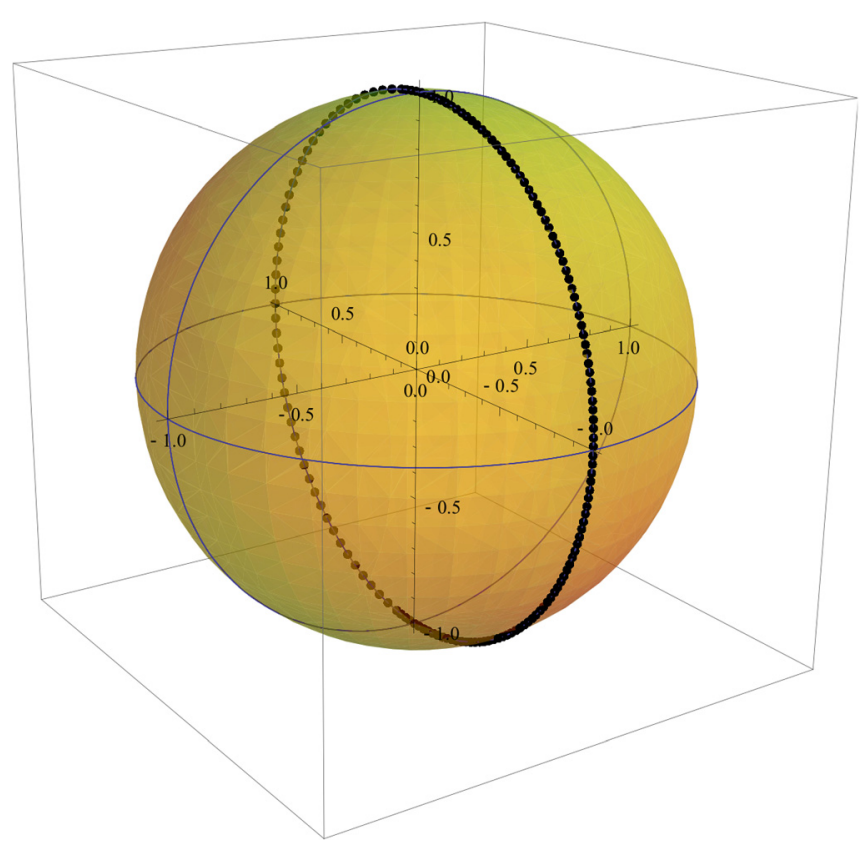

Fig. 3. Theoretical polarization states on the Poincaré sphere when the position of the observation point is varied along the $y$ direction. The parameters are the same as those of Fig. 2.

index $n=1.515$ and acute angle $\alpha=1.5^{\circ}$. The input light is linearly polarized with the azimuth at $45^{\circ}$ and wavelength $\lambda=632.8 \mathrm{~nm}$. It can be observed that the polarization state is repeated periodically along the $y$ direction.

The state of polarization can be graphically visualized by means of its representation on the Poincaré sphere. ${ }^{1}$ Remember that totally polarized states correspond to points on the surface of the Poincaré sphere, while points inside the sphere represent partially polarized light, and the center of the sphere corresponds to unpolarized light. The theoretical variation, over a period, of the polarization state on the surface of the Poincaré sphere is represented in Fig. 3 with the same values of the parameters used in Fig. 2. Note that when the observation point moves in the $y$ direction, the state of polarization goes from linearly polarized with azimuth $45^{\circ}$, to elliptically polarized following a meridian, then right-circularly polarized light is observed for a given height, then linearly polarized light with azimuth $-45^{\circ}$, followed by left-circularly polarized light, and finally linearly polarized light with azimuth $45^{\circ}$ when a full period is covered. The representation on the Poincare sphere covers nearly a meridian, and the slight differences are due to the difference between the products of the corresponding transmission coefficients $t_{\perp} t_{\perp}^{\prime}$ and $t_{\|} t_{\|}^{\prime}$.

\section{EXPERIMENT}

In order to check the predicted behavior for the polarization pattern at the output of the biprism along the $y$ direction, the experimental set-up shown schematically in Fig. 4 was developed. Note that only common optical elements that can be found in an undergraduate optics laboratory are used. Two different polarization state analyzers are employed: the first one consisting of a quarter-wave phase QWP plate, a linear polarizer $\mathrm{P}_{3}$, and a CCD camera (shown in Fig. 4) and a second one consisting of a commercial polarimeter with a pinhole at its entrance (not shown in Fig. 4).

For the incident light, a $1.5 \mathrm{~mW} \mathrm{He}-\mathrm{Ne}$ laser linearly polarized at $45^{\circ}$ has been used. The microscope objective MO together with the pinhole $\mathrm{PH}$ and lens $\mathrm{L}_{1}$ are used to spatially filter and expand the laser beam. This expanded beam impinges onto two polarizers with their transmission axes orthogonal to each other (along horizontal, $\mathrm{P}_{1}$, and vertical, $\mathrm{P}_{2}$, directions, respectively). The dividing line of the two polarizers is exactly placed in the biprism symmetry plane. A BK7 glass biprism with acute angle $\alpha=1.5^{\circ}$ and refractive index $n=1.515$ at $632.8 \mathrm{~nm}$ is used. Note that possible Fabry-Perot interferences due to multiple reflections between the polarizers output plane and biprism input plane are avoided if these two planes are not perfectly parallel. Additionally, the reflectance in these two planes is small enough to be neglected when compared to the directly transmitted laser beam.

In the first experiment, the Stokes parameters are measured as follows. A lens $\mathrm{L}_{2}$ is used to image the observation plane on a CCD camera (Pulnix TM-765) connected to a computer. A QWP and a linear polarizer $\mathrm{P}_{3}$ are used as a polarization state analyzer. Without the QWP, two images are registered, one with the polarizer $\mathrm{P}_{3}$ transmission axis along the $x$ direction and a second one with the axis along the $y$ direction. The sum and difference of these the two images result in $S_{0}$ and $S_{1}$ parameters, respectively. The subtraction of the two images registered with the polarizer $\mathrm{P}_{3}$ transmission axis at $45^{\circ}$ and at $-45^{\circ}$ gives the $S_{2}$ Stokes parameter. Finally, by placing the QWP before the polarizer $\mathrm{P}_{3}$ and acquiring two additional images with the polarizer $\mathrm{P}_{3}$ transmission axis at $45^{\circ}$ and at $-45^{\circ}$, the fourth Stokes parameter $S_{3}$ is obtained as the difference of these two last images. The Stokes parameters vary with $y$, so the Stokes vector is calculated at each pixel and then the polarization pattern is obtained.

Note that if polarizers $P_{1}$ and $P_{2}$ are removed from the setup sketched in Fig. 4, the typical interference fringes appear over an extended region. These fringes disappear when the polarizers $\mathrm{P}_{1}$ and $\mathrm{P}_{2}$ are appropriately positioned

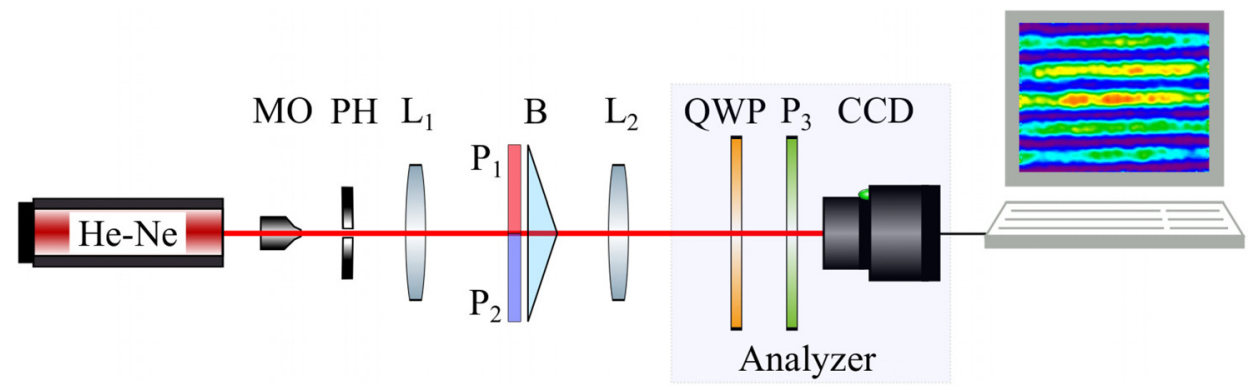

Fig. 4. Experimental set up. He-Ne: linearly polarized He-Ne laser; MO: microscope objective; $\mathrm{PH}$ : pinhole; $\mathrm{L}_{1}$, $\mathrm{L}_{2}$ : lenses; $\mathrm{P}_{1}$, $\mathrm{P}_{2}$ : linear polarizers with transmission axes orthogonally oriented; B: biprism; QWP: quarter-wave phase plate; $\mathrm{P}_{3}$ : linear polarizer; CCD: CCD camera. 


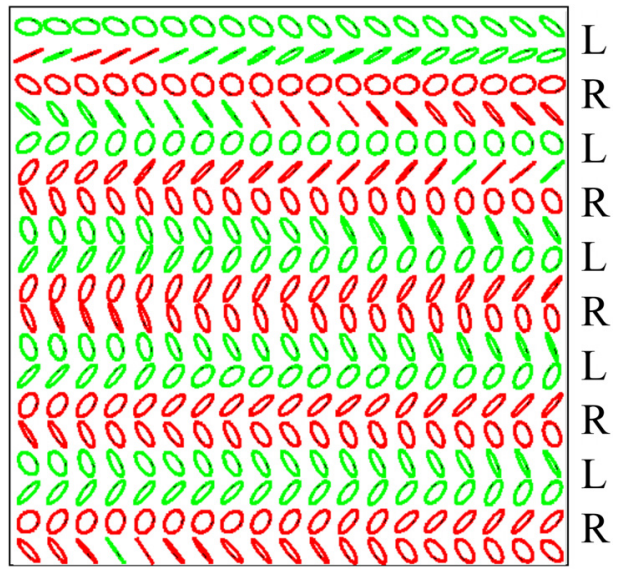

Fig. 5. Experimental polarization pattern at the observation plane measured using a CCD camera. The parameters are the same as those of Fig. 2. Labels $\mathrm{R}$ and $\mathrm{L}$ represent right-handed and left-handed polarized light, respectively.

because two fields with orthogonal polarizations are superposed on the observation plane. However, when polarizer $\mathrm{P}_{3}$ is included, fringes with a different intensity appear behind $\mathrm{P}_{3}$ due to the variation of the polarization state along the $y$ direction. This fact can be directly observed in the image shown on the computer screen of Fig. 4.

The measured polarization pattern is shown in Fig. 5. It can be observed that the polarization state changes in a periodic way along the $y$ direction, while it is almost invariant along the $x$ direction. This polarization pattern is qualitatively the same as the theoretical pattern (Fig. 2). The slight differences are mainly due to the discretization of representing the polarization ellipses, the nonuniformity of the experimental intensity, and the misalignment of polarizers $\mathrm{P}_{1}$ and $\mathrm{P}_{2}$ in the experimental setup.

In the second experiment, the polarization state analyzer and the CCD camera in the setup represented in Fig. 4 are replaced by a commercial polarimeter (Thorlabs TXP polarimeter). This polarimeter has been modified by inserting a $300 \mu \mathrm{m}$ diameter pinhole in its entrance to select an area small enough where the polarization state can be considered almost uniform. The polarimeter has been mounted on a micropositioner that can move along the $x$ and $y$ directions. When the polarimeter moves along the $x$ direction, no changes in the polarization state are observed. However, when the micropositioner moves the polarimeter along the $y$ direction, the state of polarization of the output beam changes periodically. The results are shown in Fig. 6. In this figure, it can be seen that the polarization state changes from almost linearly polarized to near circular polarization, passing through elliptical polarization and changing from left to right-handed polarization. It has also been verified that no changes in the polarization state are observed when the two polarizers $\mathrm{P}_{1}$ and $\mathrm{P}_{2}$ are removed and the polarimeter moves in any direction.

It can be observed that in half of a meridian $\left(s_{2}<0\right)$, the azimuth is constant and equal to $-\pi / 4$, while in the other half of the meridian $\left(s_{2}>0\right)$, the azimuth is constant and equal to $\pi / 4$. On the other hand, the ellipticity varies linearly from $-\pi / 4$ for left-handed circularly polarized light to $\pi / 4$ for right-handed circularly polarized light and vice versa over a full period. The experimental points are quite close to the predicted ones. The deviations observed in the azimuth are mainly due to the difference between the intensities at the output of polarizers $\mathrm{P}_{1}$ and $\mathrm{P}_{2}$. Note that the incident field is approximately a Gaussian beam instead of a plane wave with uniform intensity across the transverse section. If the Gaussian beam axis is not perfectly aligned with the biprism symmetry plane, the output fields from the polarizers $\mathrm{P}_{1}$ and $\mathrm{P}_{2}$ are not symmetric giving rise to other states of polarization than those theoretically predicted. Moreover, misalignments in the experimental setup will also affect the measured polarization states and make them deviate from the theoretical predictions.

\section{CONCLUSION}

The subject of non-uniformly polarized fields and interference with polarized light is introduced to physics students through an experiment that can be carried out in an undergraduate optics laboratory. By placing two linear polarizers with their transmission axes orthogonal to each other in front of a Fresnel biprism, it can be observed on a screen that the interference fringes disappear. This is a straightforward way to confirm some of the Fresnel-Arago laws of interference. However, students can go further and analyze what the polarization state of the field is in the superposition area. Under certain approximations, it is an easy task to find analytic expressions for the Jones vector and for the Stokes parameters of the field. The dependence of these vectors on a
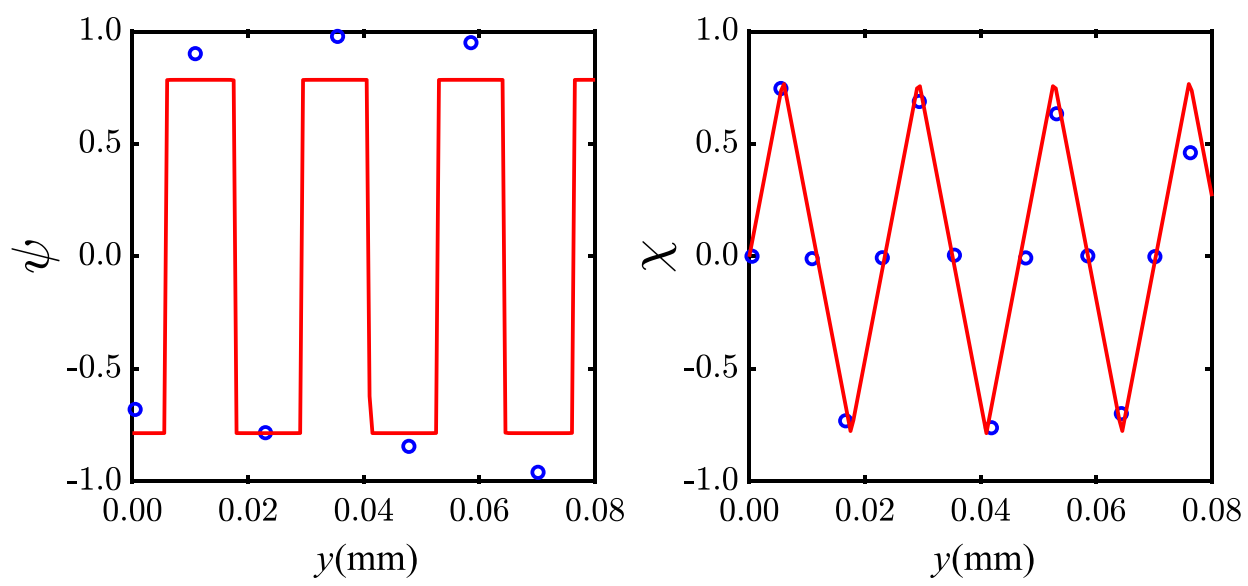

Fig. 6. Theoretical results of Eqs. (18)-(20) (solid line) and experimental measurements with the polarimeter (dots) of the azimuth (left) and ellipticity (right). The biprism parameters are the same as those of Fig. 2. 
transverse coordinate indicates that a non-uniform polarization pattern is formed in the superposition area. It has been shown that this polarization pattern can be determined experimentally, either through intensity measurements with a CCD camera or by using a commercial polarimeter.

\section{ACKNOWLEDGMENT}

This work has been supported by Spanish Ministerio de Economía y Competitividad, Project No. FIS2016-75147.

${ }^{\text {a)} E l e c t r o n i c ~ m a i l: ~ p i q u e r o @ u c m . e s ~}$

${ }^{1}$ M. Born and E. Wolf, Principles of Optics, 6th (corrected) ed. (Cambridge U.P., Cambridge, 1980).

${ }^{2}$ F. Gori, "Polarization basis for vortex beams," J. Opt. Soc. Am. A 18, 1612-1617 (2001).

${ }^{3}$ G. Piquero and J. Vargas-Balbuena, "Non-uniformly polarized beams across their transverse profiles: An introductory study for undergraduate optics courses," Eur. J. Phys. 25, 793-800 (2004).

${ }^{4}$ V. Ramírez-Sánchez, G. Piquero, and M. Santarsiero, "Generation and characterization of spirally polarized fields," J. Opt. A: Pure Appl. Opt. 11, 085708 (2009).

${ }^{5}$ Q. Zhan, "Cylindrical vector beams: from mathematical concepts to applications," Adv. Opt. Photon. 1, 1-57 (2009).

${ }^{6}$ T. G. Brown and Q. Zhan, "Focus issue: Unconventional polarization states of light," Opt. Express 18, 10775-10776 (2010).

${ }^{7}$ A. M. Beckley, T. G. Brown, and M. A. Alonso, "Full Poincaré beams," Opt. Express 18, 10777-10785 (2010).

${ }^{8}$ E. J. Galvez, S. Khadka, W. H. Schubert, and S. Nomoto, "Poincaré-beam patterns produced by nonseparable superpositions of Laguerre-Gauss and polarization modes of light," Appl. Opt. 51, 2925-2934 (2012).

${ }^{9}$ J. A. Jones, A. J. D’Addario, B. L. Rojec, G. Milione, and E. J. Galvez, "The Poincaré-sphere approach to polarization: Formalism and new labs with Poincaré beams," Am. J. Phys. 84, 822-835 (2016).

${ }^{10}$ B. Pérez-García, C. López-Mariscal, R. I. Hernández-Aranda, and J. C. Gutiérrez-Vega, "On-demand tailored vector beams," Appl. Opt. 56, 6967-6972 (2017).

${ }^{11}$ H. Rubinsztein-Dunlop, A. Forbes, M. V. Berry, M. R. Dennis, D. L. Andrews, M. Mansuripur, C. Denz, C. Alpmann, P. Banzer, T. Bauer, E. Karimi, L. Marrucci, M. Padgett, M. Ritsch-Marte, N. M. Litchinitser, N. P. Bigelow, C. Rosales-Guzmán, A. Belmonte, J. P. Torres, T. W. Neely, M. Baker, R. Gordon, A. B. Stilgoe, J. Romero, A. G. White, R. Fickler, A. E. Willner, G. Xie, B. McMorran, and A. M. Weiner, "Roadmap on structured light," J. Opt. 19, 013001 (2017).

${ }^{12}$ S. K. Mohanty, K. D. Rao, and P. K. Gupta, "Optical trap with spatially varying polarization: Application in controlled orientation of birefringent microscopic particle(s)," Appl. Phys. B 80, 631-634 (2005).

${ }^{13}$ W. Cui, F. Song, F. Song, D. Ju, and S. Liu, "Trapping metallic particles under resonant wavelength with $4 \pi$ tight focusing of radially polarized beam," Opt. Express 24, 20062-20068 (2016).

${ }^{14}$ F. Gori, "Measuring Stokes parameters by means of a polarization grating," Opt. Lett. 24, 584-586 (1999).

${ }^{15}$ O. Arteaga, R. Ossikovski, E. Kuntman, M. A. Kuntman, A. Canillas, and E. Garcia-Caurel, "Mueller matrix polarimetry on a Young's double-slit experiment analog," Opt. Lett. 42, 3900-3903 (2017).

${ }^{16}$ J. C. G. de Sande, M. Santarsiero, and G. Piquero, "Spirally polarized beams for polarimetry measurements of deterministic and homogeneous samples," Opt. Lasers Eng. 91, 97-105 (2017).

${ }^{17}$ S. Nolte, C. Momma, G. Kamlage, A. Ostendorf, C. Fallnich, F. von Alvensleben, and H. Welling, "Polarization effects in ultrashort-pulse laser drilling," Appl. Phys. A 68, 563-567 (1999).

${ }^{18}$ M. Meier, V. Romano, and T. Feurer, "Material processing with pulsed radially and azimuthally polarized laser radiation," Appl. Phys. A 86, 329-334 (2007).

${ }^{19}$ S. Matsusaka, Y. Kozawa, and S. Sato, "Micro-hole drilling by tightly focused vector beams," Opt. Lett. 43, 1542-1545 (2018).

${ }^{20} \mathrm{~J}$. Serna and G. Piquero, "Beam moments and angular momentum in nonuniformly polarized beams," Opt. Commun. 282, 1973-1975 (2009).

${ }^{21}$ E. Karimi, S. Slussarenko, B. Piccirillo, L. Marrucci, and E. Santamato, "Polarization-controlled evolution of light transverse modes and associated Pancharatnam geometric phase in orbital angular momentum," Phys. Rev. A 81, 053813 (2010).
${ }^{22}$ M. Alonso, G. Piquero, and J. Serna, "Proposals for the generation of angular momentum from non-uniformly polarized beams," Opt. Commun. 285, 1631-1635 (2012).

${ }^{23}$ D. Maluenda, A. Carnicer, R. Martínez-Herrero, I. Juvells, and B. Javidi, "Optical encryption using photon-counting polarimetric imaging," Opt. Express 23, 655-666 (2015).

${ }^{24}$ N. A. Rubin, A. Zaidi, M. Juhl, R. P. Li, J. B. Mueller, R. C. Devlin, K. Leósson, and F. Capasso, "Polarization state generation and measurement with a single metasurface," Opt. Express 26, 21455-21478 (2018).

${ }^{25}$ G. Piquero, L. Monroy, M. Santarsiero, M. Alonzo, and J. C. G. de Sande, "Synthesis of full Poincaré beams by means of uniaxial crystals," J. Opt. 20, 065602 (2018).

${ }^{26}$ F. Gori, M. Santarsiero, and R. Borghi, "Vector mode analysis of a Young interferometer," Opt. Lett. 31, 858-860 (2006).

${ }^{27}$ T. Setälä, J. Tervo, and A. T. Friberg, "Stokes parameters and polarization contrasts in Young's interference experiment," Opt. Lett. 31, 2208-2210 (2006).

${ }^{28}$ A. Luis, "Ray picture of polarization and coherence in a Young interferometer," J. Opt. Soc. Am. A 23, 2855-2860 (2006).

${ }^{29}$ M. Santarsiero, "Polarization invariance in a Young interferometer," J. Opt. Soc. Am. A 24, 3493-3499 (2007).

${ }^{30}$ J. Tervo, P. Réfrégier, and A. Roueff, "Minimum number of modulated Stokes parameters in Young's interference experiment," J. Opt. A: Pure Appl. Opt. 10, 055002 (2008).

${ }^{31}$ R. Martínez-Herrero and P. M. Mejías, "Maximizing Young's fringe visibility under unitary transformations for mean-square coherent light," Opt. Express 17, 603-610 (2009).

${ }^{32}$ Y. Li, X.-L. Wang, H. Zhao, L.-J. Kong, K. Lou, B. Gu, C. Tu, and H.-T. Wang, "Young's two-slit interference of vector light fields," Opt. Lett. 37, 1790-1792 (2012).

${ }^{33}$ M. Alonzo, M. Santarsiero, and F. Gori, "Maximizing Young fringe visibility with a universal SU2 polarization gadget," Opt. Lett. 43, 2844-2847 (2018).

${ }^{34}$ H. Partanen, B. J. Hoenders, A. T. Friberg, and T. Setälä, "Young's interference experiment with electromagnetic narrowband light," J. Opt. Soc. Am. A 35, 1379-1384 (2018).

${ }^{35}$ W. R. Mellen, "Interference of linearly polarized light with perpendicular polarizations,” Am. J. Phys. 30, 772-772 (1962).

${ }^{36}$ R. Hanau, "Interference of linearly polarized light with perpendicular polarizations," Am. J. Phys. 31, 303-304 (1963).

${ }^{37}$ J. L. Hunt and G. Karl, "Interference with polarized light beams," Am. J. Phys. 38, 1249-1250 (1970).

${ }^{38}$ D. Pescetti, "Interference between elliptically polarized light beams," Am. J. Phys. 40, 735-740 (1972).

${ }^{39} \mathrm{M}$. Henry, "Fresnel-arago laws for interference in polarized light: A demonstration experiment," Am. J. Phys. 49, 690-691 (1981).

${ }^{40}$ P. Andrés, A. Pons, and J. Ojeda-Castañeda, "Young's experiment with polarized light: Properties and applications," Am. J. Phys. 53, 1085-1088 (1985).

${ }^{41}$ S. Mallick, "Interference with polarized light," Am. J. Phys. 41, 583-584 (1973).

${ }^{42}$ J. L. Ferguson, "A simple, bright demonstration of the interference of polarized light,” Am. J. Phys. 52, 1141-1142 (1984).

${ }^{43}$ E. F. Carr and J. P. McClymer, "A laboratory experiment on interference of polarized light using a liquid crystal,” Am. J. Phys. 59, 366-367 (1991).

${ }^{44}$ B. M. Rodríguez-Lara and I. Ricardez-Vargas, "Interference with polarized light beams: Generation of spatially varying polarization," Am. J. Phys. 77, 1135-1143 (2009).

${ }^{45}$ D. Gossman, B. Pérez-García, R. I. Hernández-Aranda, and A. Forbes, “Optical interference with digital holograms," Am. J. Phys. 84, 508-516 (2016).

${ }^{46} \mathrm{G}$. R. Fowles, Introduction to Modern Optics, 2nd ed. (Holt, Rinehart, and Winston, New York, 1975).

${ }^{47}$ Y. Gorodetski, G. Biener, A. Niv, V. Kleiner, and E. Hasman, "Space-variant polarization manipulation for far-field polarimetry by use of subwavelength dielectric gratings," Opt. Lett. 30, 2245-2247 (2005).

${ }^{48}$ J. C. G. de Sande, M. Santarsiero, G. Piquero, and F. Gori, "Longitudinal polarization periodicity of unpolarized light passing through a double wedge depolarizer," Opt. Express 20, 27348-27360 (2012).

${ }^{49}$ M. Santarsiero, J. C. G. de Sande, G. Piquero, and F. Gori, "Coherencepolarization properties of fields radiated from transversely periodic electromagnetic sources," J. Opt. 15, 055701 (2013).

${ }^{50}$ G. Piquero, J. M. Movilla, P. M. Mejías, and R. Martínez-herrero, "Degree of polarization of non-uniformly partially polarized beams: a proposal," Opt. Quantum Electron. 31, 223-226 (1999). 